\title{
Effects of dexmedetomidine on inflammatory responses in patients undergoing major abdominal surgeries
}

\author{
Prof. Abdelrahman Hassan Abdelrahman, Prof. Zienab Diab, Dr. Raafat Ahmed \\ Salem, Islam Mokhtar Ahmed.
}

\begin{abstract}
Background: Surgical injury to tissue as in major abdominal surgeries causes a variety of profound inflammatory response which may lead to postoperative complications with significant co morbidity. Dexmedetomidine, a highly selective $\alpha 2-$ adrenoceptor agonist, is widely used in clinical anaesthesia, and intensive care unit. The aim of this study is to evaluate the anti inflammatory effect of dexmedetomidine in major abdominal surgeries.
\end{abstract}

Patients and methods: 40 patients for radical cystectomy in the age range of $40-65$ years participated in this prospective, randomized, double blinded, placebo controlled clinical study. Patients were randomly assigned to receive either Dexmeditomidine (Dex group) or normal saline (0.9\%) (Control group). Serum tumor necrosis factor (TNF- $\alpha$ ), Interleukin-6 (IL-6), glucose, cortisol, and C-reactive protein (CRP) were analyzed before the start of the study drug infusion, after complete recovery and the day one postoperatively.

Results: inflammatory markers were significantly higher in both groups compared to baseline measurements. TNF- $\alpha$, IL-6, glucose, and cortisol measurements were less in Dex group in postoperative period.

Conclusion: Dexmedetomidine is effective in attenuating the postoperative rise of the proinflammatory cytokine interleukin- 6 and tumor necrosis factor (TNF- $\alpha$ ) and resulted in lower levels of markers of stress response to surgery as cortisol and blood glucose.

\section{Introduction}

Surgical injury to tissue as in major abdominal surgeries causes a variety of profound physiologic reactions which are essential to the restoration of an organisms' homeostasis. The response involves a surge of stress hormones (i.e. $\mathrm{C}$ - reactive protein (CRP), cortisol, catecholamines), activation of the complement system, migration of leukocytes to the site of injury, the release of cytokines (e.g. interleukins, tumour necrosis factor) as well as other cellular products (i.e. superoxide radicals, proteases, growth factors). (Homburger JA et al, 2006).

Inflammatory response may lead to postoperative complications in the elderly, neonates, and patients with significant co morbidity. (Westaby et al, 2001).

Dexmedetomidine, a highly selective $\alpha 2$-adrenoceptor agonist, is widely used in clinical anesthesia, intensive care unit (ICU) management and pain treatment as a sedative agent. (Xianbao et al, 2013).

It is well documented that dexmedetomidine (DEX) inhibits the neuroendocrine and inflammatory response in various experimental as well as clinical settings. (Goldfarb et al, 2011).

Aim of the study:-

The aim of this study is to evaluate the anti inflammatory effect of dexmedetomidine in major abdominal or pelvi abdominal surgeries.

Patients and methods:

This prospective, randomized, double blinded, placebo controlled clinical study was approved by the Ethics and Research Committee of Sohag Faculty of Medicine, Sohag University. It was conducted at Sohag University 
Hospital between August 2015 and December 2016. Written informed consent was obtained from each patient preoperatively. The study included 40 patients in the age range of $40-65$ years.

The Inclusion criteria were as follows:-

Patients with an American Society of Anesthesiologists (ASA) physical status of I or II and scheduled for major abdominal or pelvi-abdominal surgery under general anesthesia were included in this study.

Exclusion criteria included the followings:-

A history of drug or alcohol abuse, allergy to any of the study medications , 2nd or 3rd degree heart block, treatment with $\alpha 2$ agonists or antagonists, chronic use of antipsychotic medications, chronic use of anti-inflammatory drugs, labile hypertension, cardiac dysrhythmias, coronary artery disease, renal, hepatic or cognitive impairment.

Patients were randomly assigned to receive either Dexmeditomidine (Dex group) or normal saline $(0.9 \%)$ (Control group). Randomization and enrollment were done using sequentially numbered closed envelops. All patients assessment were performed by an anesthetist blinded to the drug used for the study and to reduce the selection and pretest biases another anesthetist prepared the study drug and wrapped the syringe pumps and tubing.

All patients were premeditated with atropine (0.02 mg IM) and midazolam $5 \mathrm{mg}$ IM. Standard moniroring were applied to all patients. Two large bore intravenous cannulas were inserted in the right and left forearms.

After complete aseptic conditions central venous line was inserted in the right internal jugular or subclavian vein using Seldinger's technique for rapid infusion of fluids if needed.
Radial artery on either side was cannulated after doing Allen's test (to make sure that there is adequate blood supply for the wrist and hand by the ulnar artery) for continuous blood pressure monitoring.

Patients were randomly divided into two groups:-

Patients in the dexmedetomidine group (Dex group) received a loading dose of dexmedetomidine at $1 \mu \mathrm{g} / \mathrm{kg}$ in $20 \mathrm{ml}$ of normal saline infused IV over 10 minutes followed by a maintenance infusion of $0.5 \mu \mathrm{g} / \mathrm{kg} / \mathrm{h}$ till the end of surgery, and patients in the control group received $20 \mathrm{ml}$ of normal saline infused IV over 10 minutes followed by a maintenance infusion of normal saline till the end of surgery.

Induction of general anesthesia in both groups was done by propofol (1.5-2 $\mathrm{mg} / \mathrm{kg})$ and fentanyl (2-5 $\mu \mathrm{g} / \mathrm{kg})$. Rocuronium (1 $\mathrm{mg} / \mathrm{kg})$ was used to facilitate endotracheal intubation. Patients were ventilated with an oxygen - air mixture $(\mathrm{FiO} 2=0.4)$ with EtCO2 stabilized at $30-35 \mathrm{mmHg}$.

Anesthesia was maintained by inhalational route with Sevoflourane in both groups. All patients in both groups received ondansetron $4 \mathrm{mg}$ prior to the end of surgery.

Patients were awakened and extubated in the operative room and were transferred to the PACU upon following simple commands.

Data collection and measurements:-

Patient characteristics including age, sex, weight, ASA physical status, volume of blood loss and volume of blood transfused in addition to duration of anesthesia (time from the start of induction of anesthesia till discontinuation of inhalational anesthesia, reversal of muscle relaxant and tracheal extubation). And duration of surgery (time from the skin incision till the end of skin closure) were recorded. 
SOHAG MEDICALJOURNAL

Vol. 21 No.2 July 2017
Effects of dexmedetomidine on inflammatory responses

Islam Mokhtar Ahmed.et al
Laboratory investigations:

About $10 \mathrm{ml}$ venous blood was withdrawn before the start of the study drug infusion, after complete recovery and the day one postoperatively at 8 am from patients and control by a clean venipuncture and immediately delivered into3 serum separator tubes.

The following investigations were performed:

Enzyme $\quad-\quad$ linked

immunosorbent assay (ELISA) for the detection of serum level of the pro- inflammatory cytokines: tumor necrosis factor (TNF- $\alpha)$ and Interleukin-6 (IL-6) performed by (Assaypro, 3400 Harry S Truman Blvd St. Charles, MO 63301, USA).

Data are presented as mean \pm standard deviation or number (\%). Independent t-test and Mann Whitney tests were used to compare between study groups. Categorical data were compared by chi squared and fisher exact tests. P-value less than 0.05 was considered significant

\section{Results}

A total of forty patients were enrolled in this prospective study; twenty patients received dexmedetomidine and twenty patients as control.

All patients underwent radical cystectomy with orthotopic diversion. Patients' demographic data, duration of anesthesia and surgery, volume of blood loss, and volume of blood transfusion did not differ between the two groups (Table 1).

Table (1): Patients demographic data, duration of anesthesia and surgery, volume of blood loss, and volume of blood transfusion.

\begin{tabular}{|c|c|c|c|}
\hline & $\begin{array}{c}\text { Dex group } \\
(\mathrm{N}=20)\end{array}$ & $\begin{array}{c}\text { Control group } \\
(\mathrm{N}=20)\end{array}$ & *P-value \\
\hline Age (years) & $54.45 \pm 8.9$ & $54.65 \pm 8.5$ & 0.94 \\
\hline $\begin{array}{c}\text { Sex: } \\
\text { Male }\end{array}$ & $\begin{array}{c}18(90) \\
2(10 \%)\end{array}$ & $\begin{array}{c}17(85 \%) \\
3(15 \%)\end{array}$ & 0.4 \\
\hline $\begin{array}{c}\text { Female } \\
\text { Weight (kg) }\end{array}$ & $78 \pm 10.05$ & $79.5 \pm 7.76$ & 0.6 \\
\hline $\begin{array}{c}\text { Physical status (ASA): } \\
\text { II }\end{array}$ & $\begin{array}{c}12(40 \%) \\
\text { (m) }\end{array}$ & $\begin{array}{c}9(45 \%) \\
11(55 \%)\end{array}$ & 0.23 \\
\hline $\begin{array}{c}\text { Duration of anesthesia (hours) } \\
\text { Duration of surgery (hours) }\end{array}$ & $5.1 \pm 1.65$ & $5.28 \pm 1.45$ & 0.45 \\
\hline Volume of blood loss (ml) & $1237.5 \pm 884.6$ & $1030 \pm 542$ & 0.57 \\
\hline Volume of blood transfusion & $940 \pm 857.4$ & $975.25 \pm 1070.6$ & 0.91 \\
\hline
\end{tabular}

Data are presented as mean \pm standard deviation or number $(\%)$.

Dex group $=$ dexmedetomidine group.

*P-value denotes statistical significance.

The plasma level of TNF- $\alpha$, IL- 6 , serum cortisol, blood glucose, and C-reactive protein before the start of the study drug infusion, after complete recovery and the day one postoperatively at 8 am were presented in tables $2,3,4,5,6$ and figures $1,2,3,4,5$ respectively. 


\section{Tumor necrosis factor $-\alpha$ (TNF- $\alpha)(\mathrm{pg} / \mathrm{ml})$ :}

Table 2 and figure 1 showed that tumour necrotic factor was significantly lower in Dex group than control group at recovery and the day one postoperatively at 8 am.

TNF- $\alpha$ was significantly lower at complete recovery when compared with the baseline value in both groups.

TNF- $\alpha$ was significantly lower in the day one postoperatively at 8 am when compared with the baseline value in the control group.

Table (2): Tumor necrosis factor $-\alpha(\mathrm{TNF}-\alpha)$.

\begin{tabular}{|c|c|c|c|}
\hline & $\begin{array}{c}\text { Dex group } \\
\mathrm{N}=20\end{array}$ & $\begin{array}{c}\text { Control group } \\
\mathrm{N}=20\end{array}$ & P-value \\
\hline $\begin{array}{c}\text { TNF- } \alpha \text { before the start of } \\
\text { the study drug infusion } \\
\text { (baseline) }\end{array}$ & $16.4 \pm 2.1$ & $17.4 \pm 2.6$ & 0.184 \\
\hline $\begin{array}{c}\text { TNF- } \alpha \text { at recovery } \\
\text { TNF- } \alpha \text { the day one } \\
\text { postoperatively at } 8 \text { am }\end{array}$ & $13.8 \pm 3.1$ & $\# 36 \pm 5.3$ & $<0.001^{*}$ \\
\hline
\end{tabular}

Data are presented as mean \pm standard deviation.

Dex group $=$ dexmedetomidine group.

*P-value denotes statistical significance versus the control group.

T P-value denotes statistical significance with the base line value in the Dex group.

\# p-value denotes statistical significance with the base line value in the control group.

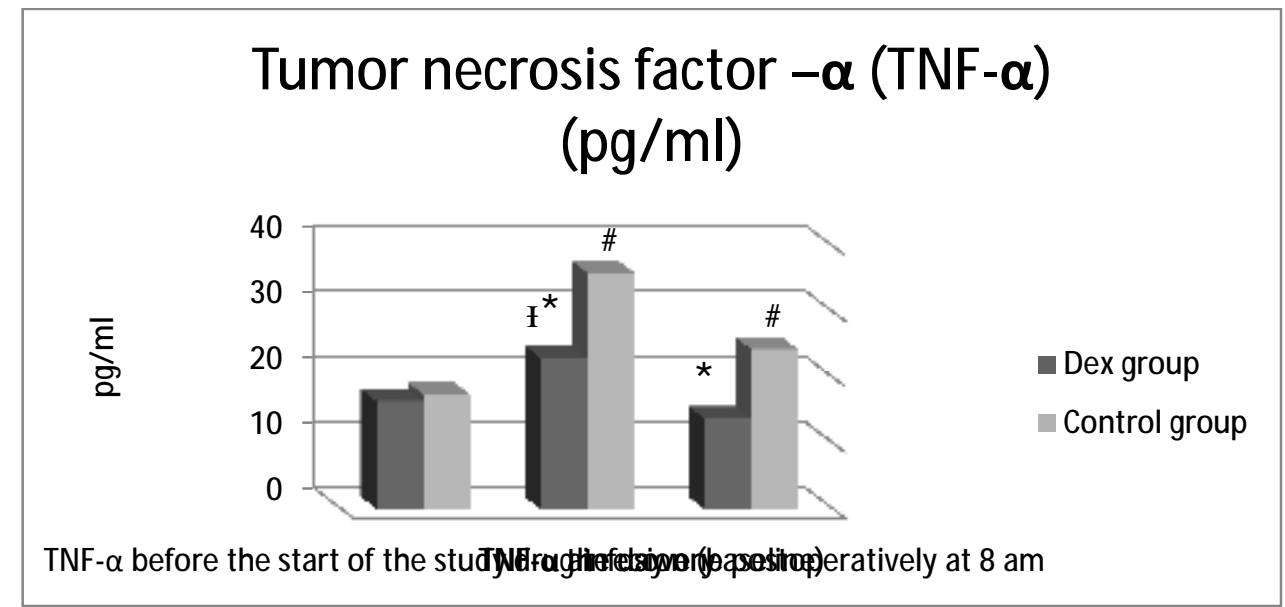

Figure (1): Tumor necrosis factor $-\alpha($ TNF- $\alpha)$.

Data are presented as mean.

Dex group $=$ dexmedetomidine group.

$* \mathrm{P}$-value $<0.05$ denotes statistical significance versus the control group.

T P-value denotes statistical significance with the base line value in the Dex group.

\# P-value denotes statistical significance with the base line value in the control group.

Serum Interleukine-6 (IL-6) (pg/ml):

Serum Interleukine-6 was significantly lower in Dex group than control group at recovery and the day one postoperatively at 8 am and serum IL-6was significantly lower at complete recovery and in day one postoperatively at 8 am when compared with the baseline value in the control group as shown in table (3) and figure (2). 
SOHAG MEDICALJOURNAL

Vol. 21 No.2 July 2017
Effects of dexmedetomidine on inflammatory responses

Islam Mokhtar Ahmed.et al

Table (3): Serum Interleukine-6 (IL-6).

\begin{tabular}{|l|c|r|r|}
\hline & $\begin{array}{c}\text { Dex group } \\
\mathrm{N}=20\end{array}$ & $\begin{array}{c}\text { Control group } \\
\mathrm{N}=20\end{array}$ & P-value \\
\hline $\begin{array}{l}\text { IL-6 before the start } \\
\text { of the study drug } \\
\text { infusion (baseline) }\end{array}$ & $8.4 \pm 1$ & $8.7 \pm 1.2$ & 0.441 \\
\hline IL-6 at recovery & $9.3 \pm 1$ & $\# 38.1 \pm 8.8$ & $<0.001^{*}$ \\
\hline $\begin{array}{l}\text { LL-6 the day one } \\
\text { postoperatively at } 8 \\
\text { am }\end{array}$ & $9.6 \pm 1.2$ & $\# 57.1 \pm 11.5$ & $<0.001^{*}$ \\
\hline
\end{tabular}

Data are presented as mean \pm standard deviation.

Dex group $=$ dexmedetomidine group.

*P-value denotes statistical significance versus the control group.

\# P-value denotes statistical significance with the baseline value in the control group.

\section{serum Interleukin-6 (IL-6)}

(pg/ml)

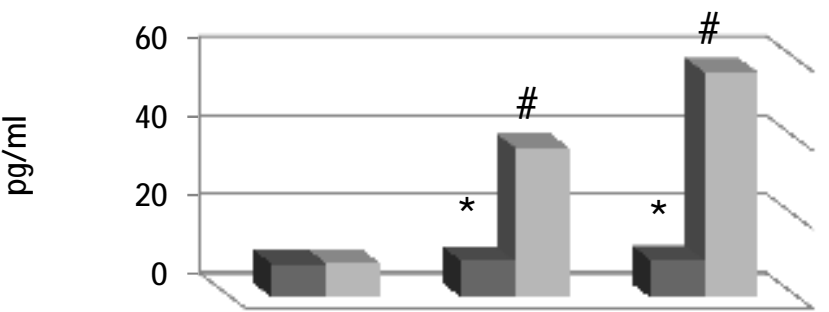

- Dex group

Control group

II-6 before the start of the study drimgerative at 8 am

Figure (2): Serum Interleukine-6 (IL-6).

Data are presented as mean.

Dex group $=$ dexmedetomidine group.

$*$ P-value $<0.05$ denotes statistical significance versus the control group.

\# P-value denotes statistical significance with the base line value in the control group.

Serum cortisol (ug/dl):

Serum cortisol was significantly lower in Dex group than study group at recovery as shown in table (4) and figure (5).

Table (4): Serum cortisol level.

\begin{tabular}{|l|c|c|c|}
\hline & $\begin{array}{c}\text { Dex group } \\
\mathrm{N}=20\end{array}$ & $\begin{array}{c}\text { Control group } \\
\mathrm{N}=20\end{array}$ & P-value \\
\hline $\begin{array}{l}\text { Serum Cortisol before the start } \\
\text { of the study drug infusion }\end{array}$ & $16.3 \pm 5.5$ & $15.3 \pm 7.9$ & 0.64 \\
\hline Serum Cortisol at recovery & $17.2 \pm 4.8$ & $22.5 \pm 7.5$ & $0.01^{*}$ \\
\hline $\begin{array}{l}\text { Serum Cortisol the day one } \\
\text { postoperatively at } 8 \text { am }\end{array}$ & $19.9 \pm 12.7$ & $19.4 \pm 5.8$ & 0.89 \\
\hline
\end{tabular}

Data are presented as mean \pm standard deviation.

Dex group $=$ dexmedetomidine group. 
*P-value denotes statistical significance versus the control group.

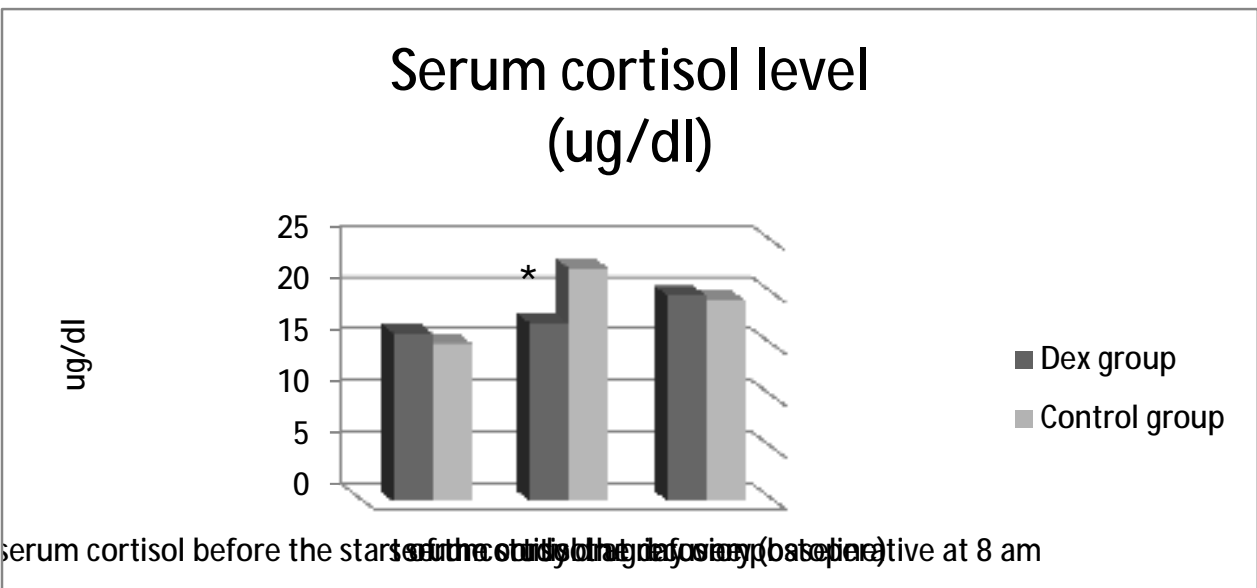

Figure (3): Serum cortisol level

Data are presented as mean.

Dex group $=$ dexmedetomidine group.

*P-value denotes statistical significance versus the control group.

Blood glucose (mg/dl):

Blood glucose was significantly lower in Dex group than study group at recovery and it was significantly lower at recovery and day one postoperatively at 8 am when compared with the baseline value in either groups as shown in table (5) and figure (4).

Table (5): Blood glucose.

\begin{tabular}{|c|c|c|c|}
\hline & $\begin{array}{l}\text { Dex group } \\
\quad \mathrm{N}=20\end{array}$ & $\begin{array}{l}\text { Control group } \\
\mathrm{N}=20\end{array}$ & P-value \\
\hline $\begin{array}{l}\text { Blood glucose before the } \\
\text { start of the study drug } \\
\text { infusion (baseline). }\end{array}$ & $98.9 \pm 29.9$ & $115.8 \pm 53.2$ & 0.2 \\
\hline $\begin{array}{l}\text { Blood glucose at } \\
\text { recovery. }\end{array}$ & Т $193.4 \pm 76.5$ & \# $271.4 \pm 118.2$ & $0.018^{*}$ \\
\hline $\begin{array}{c}\text { Blood glucose the day } \\
\text { one postoperatively at } 8 \\
\text { am }\end{array}$ & Т $176 \pm 123.2$ & \# 205.4 149.6 & 0.501 \\
\hline
\end{tabular}

Data are presented as mean \pm standard deviation.

Dex group $=$ dexmedetomidine group.

*P-value denotes statistical significance versus the control group.

T P-value denotes statistical significance with the base line value in the Dex group.

\# P-value denotes statistical significance with the base line value in the control group. 


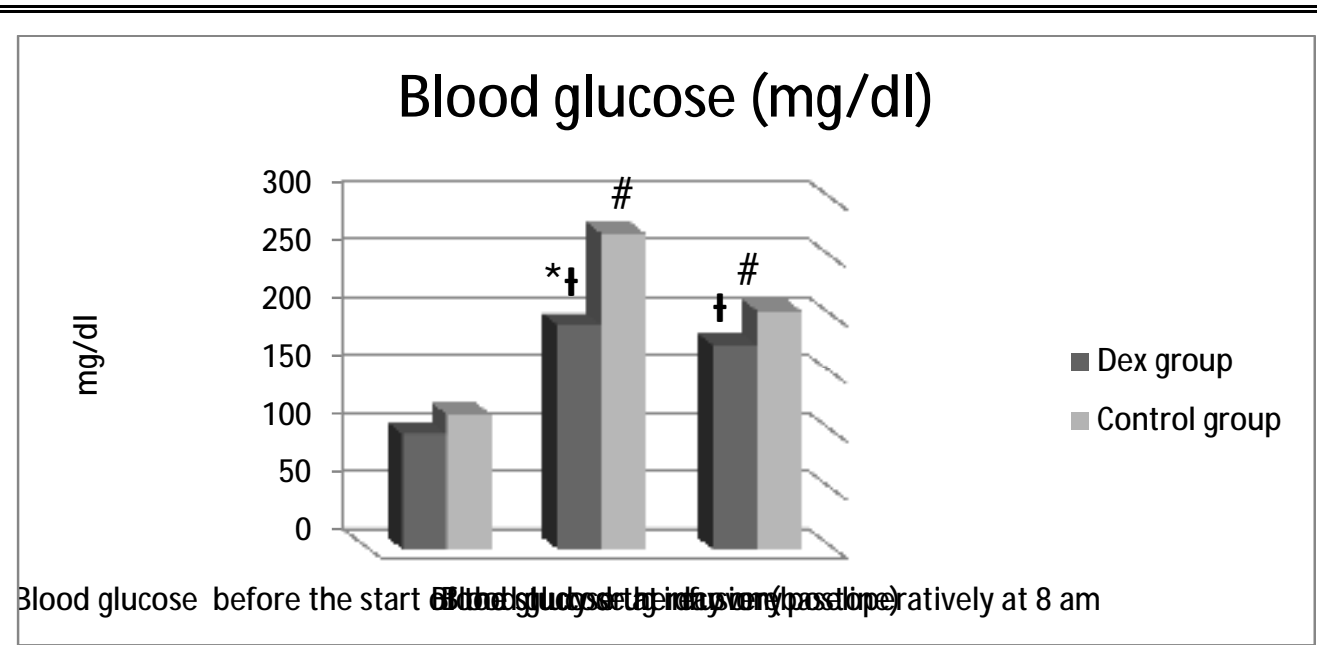

Figure (4): Blood glucose.

Data are presented as mean.

Dex group $=$ dexmedetomidine group.

*P-value $<0.05$ denotes statistical significance versus the control group.

T P-value denotes statistical significance with the base line value in the Dex group

\# P-value denotes statistical significance with the base line value in the control group.

C- Reactive protein (CRP) (mg/l):

There was no significant difference in CRP between both groups while $\mathrm{C}$-reactive protein was significantly lower in day one postoperatively at 8 am when compared with the baseline value in either groups as shown in table (6) and figure (5).

Table (6): C- reactive protein (CRP)

\begin{tabular}{|c|c|c|c|}
\hline & $\begin{array}{c}\text { Dex group } \\
\mathrm{N}=20\end{array}$ & $\begin{array}{c}\text { Control group } \\
\mathrm{N}=20\end{array}$ & P-value \\
\hline $\begin{array}{c}\text { CRP before the } \\
\text { start of the study } \\
\text { drug infusion } \\
\text { (baseline). }\end{array}$ & $3.2 \pm 6.2$ & $3.3 \pm 3.9$ & 0.980 \\
\hline CRP at recovery. & $2.5 \pm 5.4$ & $3.2 \pm 3.9$ & 0.673 \\
\hline $\begin{array}{c}\text { CRP the day one } \\
\text { postoperative at } 8 \\
\text { am. }\end{array}$ & T $12.4 \pm 8.7$ & $\# 15.3 \pm 7.6$ & 0.259 \\
\hline
\end{tabular}

Data are presented as mean \pm standard deviation.

Dex group $=$ dexmedetomidine group.

*P-value denotes statistical significance versus the control group.

T P-value denotes statistical significance with the base line value in the Dexl group.

\# P-value denotes statistical significance with the base line value in the control group. 


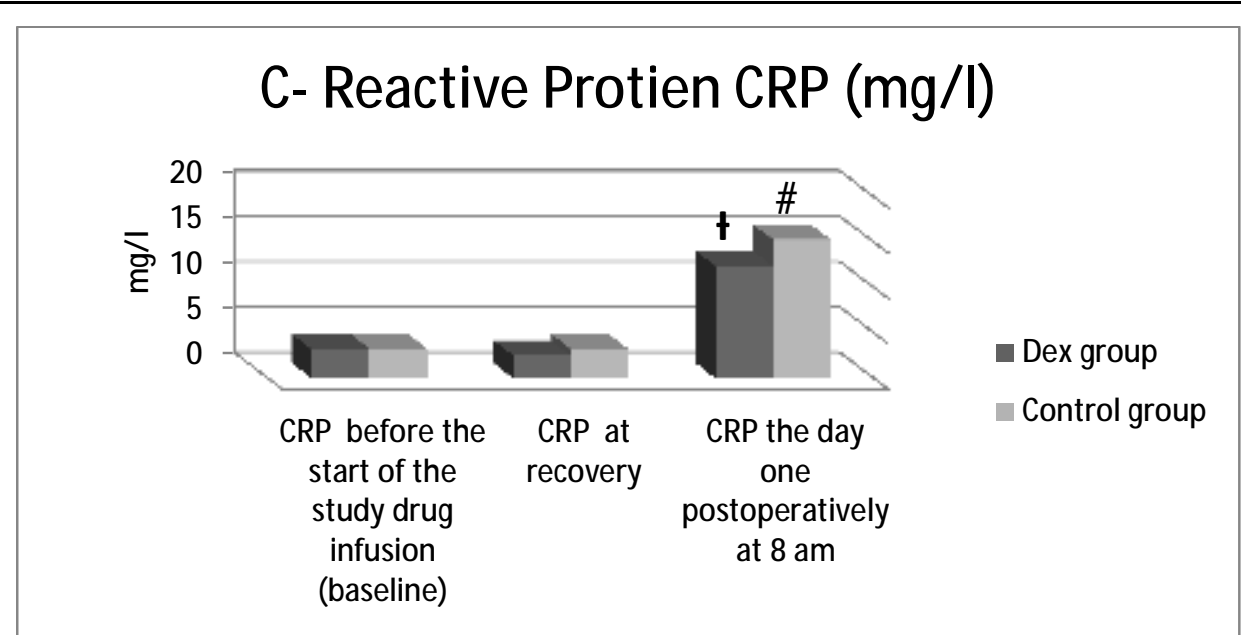

Figure (5): C-reactive protein (CRP).

Data are presented as mean.

Dex group $=$ dexmedetomidine group.

$* \mathrm{P}$-value $<0.05$ denotes statistical significance versus the control group.

T P-value denotes statistical significance with the base line value in the Dex group.

\# P-value denotes statistical significance with the base line value in the control group.

\section{Discussion}

Dexmedetomidine is a centrally acting $\alpha 2$-adrenergic agonist reducing norepinephrine turnover and decreasing central sympathetic outflow. Multiple mechanisms of dexmedetomidine action have been postulated including the modulation of cytokine production by macrophages and monocytes during the stress response which may also be stimulated via the $\alpha 2$-adrenoceptors, inhibition of apoptosis, central sympatholytic effects including the stimulation of cholinergic anti- inflammatory pathway, and anti nociceptive action involving interactions between pain and immune factors such as proinflammatory cytokines (kang et al, 2013).

The stress response to surgery is a neuroendocrine and cytokine response to surgical trauma. It is characterized by increases in catecholamine and steroid hormones, with metabolic consequences (O'Riain et al., 2005). This stress response is considered as the physiological homeostatic defence mechanism, important for the body for adaptation and developing resistance to the noxious insults. If such exaggerated physiological changes are prolonged, that may result in exhaustion of essential components of the body increasing morbidity and mortality (Velickovic et al., 2002).

In this study, patients received dexmedetomidine had significantly lower random blood glucose at recovery. Previous studies suggested that dexmedetomidine did inhibit the hyperglycaemic response to surgery significantly more than placebo, and this may reflect attenuation of the sympatho-adrenal response (Mukhtar et al., 2006).

We found no significant difference in CRP between both groups in post operative period. When compared to saline, Ueki et al found no significant difference in CRP with patients received dexmedetomidine after cardiopulmonary bypass (Ueki et al, 2014). Intraoperative Infusion of dexmedetomidine did not decrease CRP significantly after major spinal 
surgery (Bekker et al, 2013). While in laparoscopic cholecystectomy, CRP were significantly less in patients received dexmedetomidine in postoperative day one (kang et al, 2013).

Patients received dexmedetomidine had lower serum cortisol at recovery. Aho et al. found that patients receiving dexmedetomidine had significantly lower intraoperative cortisol levels as compared with those who did not receive the drug before surgery (Aho et al., 1992). Intraoperative infusion of dexmedetomidine decreased serum cortisol in major spinal surgery in post anaesthesia care unit (Bekker et al, 2013). In contrast to the present study, Aantaa et al. found no significant difference in serum cortisol in saline and dexmedetomidine groups. Their study was on patients undergoing minor gynaecologic surgery (Bekker et al, 2013).

Interleukin-6 is a proinflammatory cytokine that exerts both local and systemic effects for reducing tissue injury and combating infections while promoting biochemical mechanisms of healing and repair (Sheeran and Hall, 1997). It is the principal interleukin released after surgery and acts as a reliable indicator of the inflammatory response to surgical trauma (Jameson et al, 1997). We found that Patients received dexmedetomidine had lower serum IL-6 level at recovery and one day after surgery. These results agree with Yacout et al. who worked in patients undergoing major abdominal surgery (Yacout et al., 2012). In patients received dexmedetomidine, IL-6 concentrations declined significantly after laparoscopic cholecystectomy (Bekker et al, 2013). After cardiopulmonary bypass, plasma IL-6 levels were higher in the saline group than the dexmedetomidine group (Ueki et al, 2014). Dexmedetomidine did not differentially modulate levels of IL-6 after major spinal surgery (Bekker et al, 2013).

Tumor necrosis factor- $\alpha$ is also a proinflammatory cytokine that regulates several other cytokines (Clark, 2007).TNF was significantly lower in Patients received dexmedetomidine at recovery and one day after surgery. Perioperative use of dexmedetomidine as an adjunct to general anesthesia was found to cause significant decreases TNF- $\alpha$ within a period of 24 hours postoperatively ( $\mathbf{L i}$ et al, 2015). Tasdogan et al. showed that intravenous dexmedetomidine infusion decreases serum TNF after abdominal surgery (Tasdogan et al, 2009).

\section{Conclusion:}

Dexmedetomidine is effective in attenuating the postoperative rise of the proinflammatory cytokine interleukin6 and tumor necrosis factor (TNF- $\alpha$ ) and resulted in lower levels of markers of stress response to surgery as cortisol and blood glucose.

\section{References}

1. Aho, M, Scheinin, M, Lehtinen, A, Erkola, O. Intramuscularly administered dexmedetomidine attenuates hemodynamic and stress hormone responses to gynecologic laparoscopy. Anesth Analg 1992, 75, 932-9.

2. Bekker, A, Haile, M, Kline, R, Didehvar, S, abu, R. The effect of intraoperative infusion of dexmedetomidine on the quality of recovery after major spinal surgery. J Neurosurg Anesthesiol 2013, 25, 1624.

3. Clark, I. A. How TNF was recognized as a key mechanism of disease. Cytokine Growth Factor Rev. 18, 33543 (2007).

4. Goldfarb Y, Sorski L, Benish M, Levi B, Melamed R, Ben-Eliyahu S. Improving postoperative immune status and resistance to cancer metastasis: a 
combined perioperative approach of immunostimulation and prevention of excessive surgical stress responses. Ann Surg. 2011; 253:798-810.

5. Homburger JA, Meiler SE. Anesthesia drugs, immunity, and long- erm outcome. Curr OpinAnaesthesiol. 2006; 19:423-8.

6. Jameson, P., Desborough, J. P., Bryant, A. E. \& Hall, G. M. The effect of cortisol suppression on IL-6 and white blood cell responses to surgery. Acta Anaesthiol Scand. 41, 304-8 (1997).

7. Kang, S. H. et al. Effects of dexmedetomidine on inflammatory responses in patients undergoing laparoscopic cholecystectomy. Acta Anaesthesiol Scand. 57, 480-7 (2013).

8. Li, YY, Ge, DJ, Li, JY, and Qi, B, Sex Differences in the Morphine-Sparing Effects of Intraoperative Dexmedetomidine in PatientControlled Analgesia Following General Anesthesia: A ConsortProspective, Randomized, Controlled Clinical Trial. Medicine (Baltimore), 2016. 95(18): p. e3619.

9. Mukhtar,AM, Obayah, EM, Hassona, AM. Preliminary experience with dexmedetomidine in pediatric anesthesia. Anesth Analg 2006, 103, 250 .

10.O'Riain SC, Buggy DJ, Kerin MJ. Inhibition of the stress response to breast cancer surgery by regional anesthesia and analgesia does not affect vascular endothelial growth factor and prostaglandin E2. Anesth Analg 2005;100:1244-9.
11. Sheeran, P. \& Hall, G. M. Cytokines in anesthesia. BJA. 78, 201-19 (1997).

12.Tasdogan M, Memis D, Sut N, Yuksel M. Results of a pilot study on the effects of propofol and dexmedetomidine on inflammatory responses and intraabdominal pressure in severe sepsis. J Clin Anesth. 2009; 21:394-400.

13.Ueki, M, Kawasaka, T, Haba, K., Hamada, K., Kawazaki, C. The effects of dexmedetomidine on inflammatory mediators after cardiopulmonary bypass. Anaesthesia 2014, 69, 693-700.

14. Velickovic I, Yan J, Gross JA. Modifying the neuroendocrine stress response. Seminars in anaesthesia. Perioper Med Pain 2002;21:16-25.

15. Westaby S, Saatvedt K, White S, Katsumata T, van Oeveren W, Halligan $\mathrm{PW}$. Is there a relationship between cognitive dysfunction and systemic inflammatory response after cardiopulmonary bypass?Ann Thorac Surg. 2001; 71:667-72.

16.Xianbao, L.; Hong, Z.; Xu, Z.; Chunfang, Z.; Dunjin, C. Dexmedetomidine reduced cytokine release during postpartum bleedinginduced multiple organ dysfunction syndrome in rats. Mediat. Inflamm. 2013, 2013, 627831.

17. Yacout, A., Osman, H, Abdeldaem, M, Hammouda, S. Effect of intravenous dexmedetomidine infusion on some proinflammatory cytokines, stress hormones and recovery profile in major abdominal surgery. Alexandria Journal of Medicine 2012, 48, 3-8. 
SOHAG MEDICALJOURNAL

Vol. 21 No.2 July 2017
Effects of dexmedetomidine on inflammatory responses Islam Mokhtar Ahmed.et al 
SOHAG MEDICALJOURNAL

Vol. 21 No.2 July 2017
Effects of dexmedetomidine on inflammatory responses Islam Mokhtar Ahmed.et al 